Proc. Indian Acad. Sci. (Earth Planet. Sci.), Vol. 93, No. 1, March 1984, pp. 17-25.

C) Printed in India.

\title{
Effect of relative humidity on the scattering properties of urban aerosol particles
}

\author{
R K GOEL, N C VARSHNEYA and T S VERMA \\ Department of Physics, University of Roorkee, Roorkee 247667, India \\ MS received 8 April 1983; revised 6 October 1983
}

\begin{abstract}
The effect of increase in relative humidity on the angular variation of scattered light intensity distribution function and on asymmetry factor has been studied theoretically for three sizes of particles which are representative of urban aerosols. The increase in relative humidity causes change in physical properties of the particles, like radius $(r)$ and complex refractive index $(n-i k)$. The study is based on these changes in physical characteristics.

It has been found that there are almost negligible changes in the function $i$ at low humidities $(\leqslant 40 \%$ ) for all the three aerosol sizes studied, which may be due to insignificant changes in the physical properties. At higher values, however, the physical parameters as well as the function $i$ show significant changes. The changes in $i$ are systematic for the particle very small compared to the wavelength of incident radiation but for the particles comparable or larger it is not so. The asymmetry factor changes with the increase in relative humidity, for smaller particle it increases and for two larger particles changes are of fluctuative nature.
\end{abstract}

Keywords. Relative humidity; angular variation; scattered light intensity; urban aerosols.

\section{Introduction}

The minute suspended particles in the atmosphere, the aerosols, affect the radiation budget of the earth-atmosphere system very significantly due to their role in scattering and absorbing the incoming as well as outgoing radiation. The particles scatter the incident radiation in different directions, in varying proportions and influence the angular distribution of diffused radiation. Various aspects of the scattering properties of aerosols have been studied in detail theoretically as well as experimentally (Deirmendjian 1969; Kerker 1969; and Van De Hulst 1957).

The angular distribution of the light intensity, scattered by aerosols, can be obtained by computing the two Mie-scattering functions $i_{1}$ and $i_{2}$ which correspond to the two polarization states of the incident light. These functions depend on the size and complex refractive index of the scatterer as well as on the wavelength of the incident radiation. The functions have been computed for many types of particles (with different sizes and refractive index) using different wavelengths of the incident radiation (Denman 1966; Shifrin and Zelmanovich 1967). The relative humidity of the ambient atmosphere affects these functions as it changes the size and the complex refractive index of aerosols. An increase in relative humidity causes increase in the size and decrease in the real as well as imaginary parts of the refractive index (for the visible electromagnetic radiation) of the particle (Hänel 1976). The effect of change in the relative humidity on the physical properties and on the scattering parameters like scattering coefficient and scattering albedo etc., has been studied extensively for six different models of aerosols using radiations of different wavelength by Hänel (1976). However, nothing has been said about the change in the angular variation of the functions, $i_{1}, i_{2}$, either for a single 
particle or for a collection of particles. The angular distribution of scattered light is very important for radiation transfer studies in atmosphere and hence is discussed in detail.

The asymmetry factor $\left(G_{\mathrm{FAC}}\right)$ is calculated since it depends on the angular distribution of the scattered light. It is directly proportional to the resultant of scattered wave momentum in the forward direction and like function $i$, depends on the size parameter and complex refractive index of the scatterer.

\section{Theoretical approach}

The functions $i_{1}, i_{2}$ relate the incident light to the scattered light, as given below

$$
I=E_{0} \frac{\lambda^{2}}{4 \pi^{2}}\left[\frac{1}{2}\left(i_{1}+i_{2}\right)\right]
$$

Here, $I$ is the intensity (flux per unit solid angle) of scattered light at an angle $\theta, E_{0}$ is the irradiance (flux per unit area of cross-section) of the incident light, $\lambda$ the wavelength of the light. The function $\frac{1}{2}\left(i_{1}+i_{2}\right)$ gives the intensity distribution function for unpolarized incident light and we represent it by $i$. In actual the aerosols are irregular in shape but for theoretical purposes these are assumed to be spherical. This assumption leads to results, not very much different from the experimental observations and is widely used. The mathematical expressions for $i_{1}, i_{2}$ derived by $\mathrm{Mie}$ for spherical particles are given as

$$
\begin{aligned}
& i_{1}=\left|S_{1}\right|^{2}=\left|\sum_{n=1}^{\infty} \frac{2 n+1}{n(n+1)}\left(a_{n} \pi_{n}+b_{n} \tau_{n}\right)\right|^{2}, \\
& i_{2}=\left|S_{2}\right|^{2}=\left|\sum_{n=1}^{\infty} \frac{2 n+1}{n(n+1)}\left(a_{n} \tau_{n}+b_{n} \pi_{n}\right)\right|^{2} .
\end{aligned}
$$

Here $a_{n}, b_{n}, \pi_{n}$ and $\tau_{n}$ are the functions of radius $(r)$ and complex refractive index $(n-i k)$ of the scatterer, scattering angle $(\theta)$ and wavelength $(\lambda)$ of the incident radiation. The detailed expressions for these functions are available in many standard text books (see, for example, Van de Hulst 1957).

In the above expressions for $i_{1}$ and $i_{2}$, the series terminates at infinity and it not possible to use these for computational purpose. However, it has been found that the series practically converges for lesser number of terms, $N$, which depends on the size parameter $x(=2 \pi r / \lambda)$. The expressions used in the algorithm for $N$ are based on the observations of Khare (1976) and for different ranges of $x$ are given as,

$$
N \equiv\left[\begin{array}{ll}
x+4 x^{1 / 3}+1 & 0 \cdot 02 \leqslant x \leqslant 8 \\
x+4 \cdot 05 x^{1 / 3}+2 & 8<x<4200 \\
x+4 x^{1 / 3}+2 & 4200 \leqslant x \leqslant 20000
\end{array}\right.
$$

The asymmetry factor $\left(G_{\mathrm{FAC}}\right)$ represents the mean value of $\cos \theta$ over the scatterer weighted by the values of scattered intensity, $I(\theta)$, and is represented as

$$
\begin{aligned}
G_{\mathrm{FAC}}= & \frac{4}{x^{2} Q_{\mathrm{SCA}}} \sum_{n=1}^{N}\left[\frac{n(n+2)}{n+1} \operatorname{Re}\left(a_{n} a_{n+1}^{*}+b_{n} b_{n+1}^{*}\right)\right. \\
& \left.+\frac{2 n+1}{n(n+1)} \operatorname{Re}\left(a_{n} b_{n}^{*}\right)\right] .
\end{aligned}
$$


Here $a_{n}^{*}, b_{n}^{*}$ and $a_{n+1}^{*}$ being the complex conjugates of $a_{n}, b_{n}$ and $b_{n+1}$ respectively.

The computational work is done using the algorithm developed by Wiscombe (1979). It has been properly modified and is used on computer DEC-20 system. There are provisions for different types of particles, smaller as well larger than the wavelength of light, and accuracy of the algorithm had been verified before using for actual cases.

The data for the change in size and complex refractive index of the particles with the change in relative humidity is taken from Hänel (1976) and is tabulated in table 1 . The dry radii of the particles are $0.04472,0.1706$ and $1.017 \mu \mathrm{m}$ respectively and these were sampled by Hänel at Mainz (W. Germany). The data are for the increasing part of relative humidity cycle and all the computations are done for electromagnetic radiation of wavelength $0.55 \mu \mathrm{m}$.

\section{Observations and discussion}

The computed results are plotted in figures 1 to 4 . The simplest pattern for function $i$ is observed for the smallest particle $(r(0)=0.04472 \mu \mathrm{m})$. It is shown in figure 1 and it is clear that as the relative humidity increases, the function also increases. The changes being very small, almost negligible at low values, upto $40 \%$, of relative humidity and quite significant at higher values. The function has almost similar patterns in forward

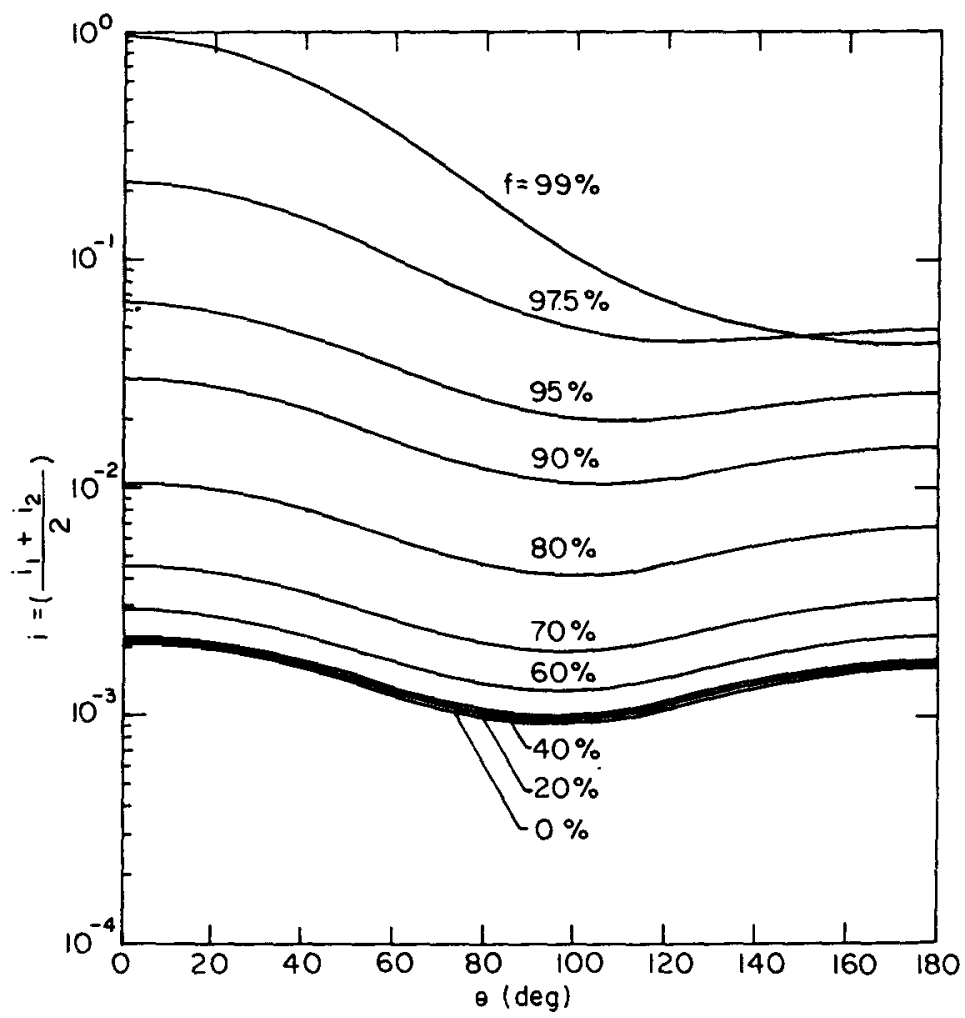

Figure 1. Angular variation of intensity distribution function at different values of relative humidity $(0 \% \leqslant f \leqslant 99 \%)$ for the particle of dry radius, $r(0)=0.04472 \mu \mathrm{m}$. 


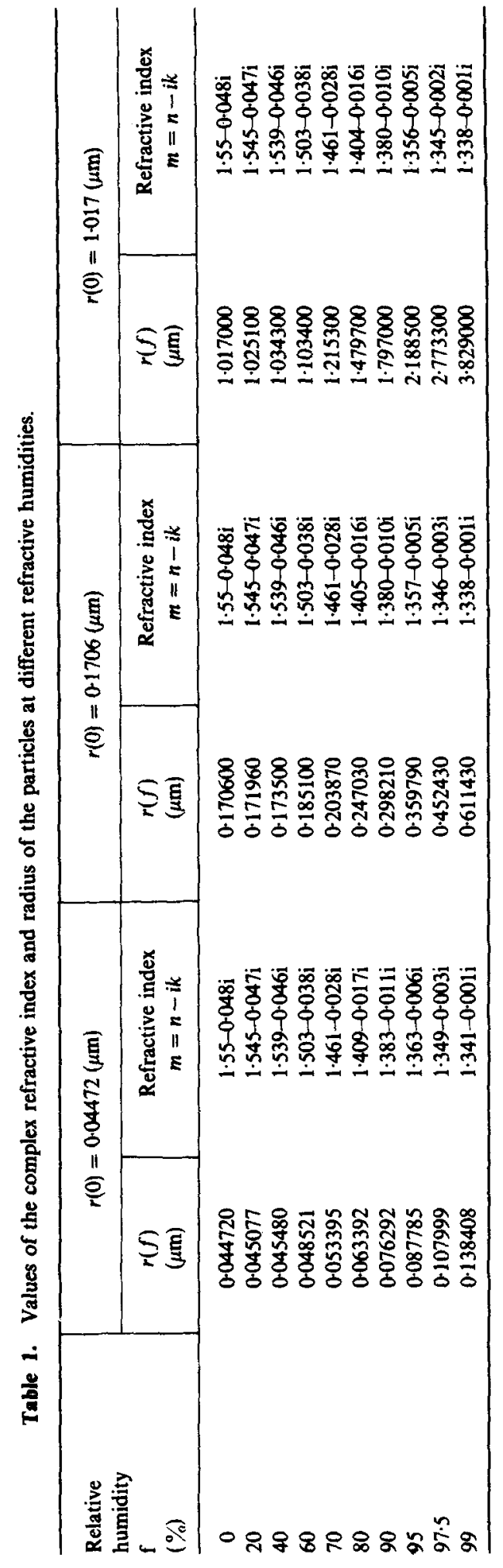




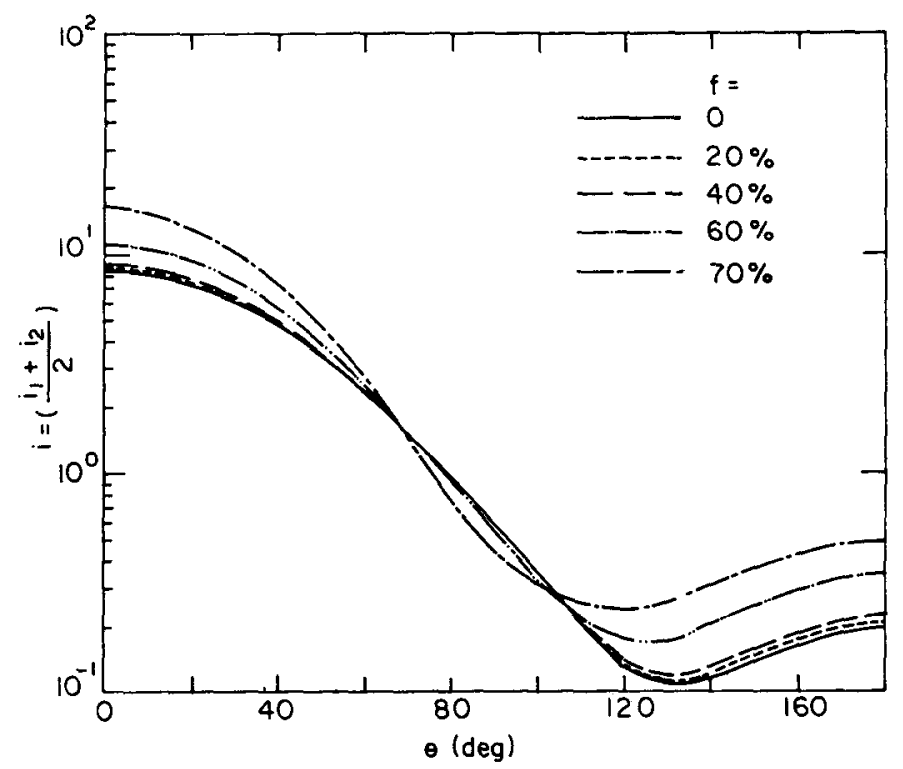

Figure 2(a). Angular variation of intensity distribution function at lower values of relative humidity $(0 \% \leqslant f \leqslant 70 \%)$ for the particle of dry radius $r(0)=0.1706 \mu \mathrm{m}$.

$\left(0^{\circ} \leqslant \theta \leqslant 90^{\circ}\right)$ and backward $\left(90^{\circ}<\theta<180^{\circ}\right)$ lobes, except at very high relative humidities $(\geqslant 95 \%)$.

The changes in angular variation of $i$ for second particle $(r(0)=0.1706 \mu \mathrm{m})$ shown in figures 2(a) and 2(b), are a bit complicated. As stated earlier, in this case also changes are negligible upto $40 \%$ relative humidity. At higher values $(\leqslant 70 \%)$ changes in the functions are significant at all the angles except the region $50^{\circ} \leqslant \theta \leqslant 110^{\circ}$. On further increasing the relative humidity, the changes are more pronounced, but these are regular and positive in a small solid angle $\left(\theta \leqslant 10^{\circ}\right)$ along the forward direction only. In the rest of the region the nature of $i$ is oscillatory, the number of oscillations depending on the relative humidity.

For the third particle $(r(0)=1.017 \mu \mathrm{m})$, as shown in figures 3(a) and 3(b), the picture is not quite clear. The angular variation for the dry particle itself has so many fluctuations. For this particle also, changes are significant only at higher $(>40 \%)$ values of relative humidity and are regular in a small solid angle $\left(\theta \leqslant 10^{\circ}\right)$ along the direction of incident beam.

The variation in the asymmetry factor, in all the three cases, is clear and easy to comprehend. Whereas for the smallest particle it increases continuously and appreciably at higher values $(>60 \%)$, for the two bigger particles it does not change much at all the values of relative humidity.

As mentioned earlier, the change in the function $i$ on increasing the relative humidity is due to change in size $(r)$ and refractive index $(n-i k)$ of the particle. For all the particles there is increase in size as the relative humidity increases, the magnitude of change depending on the chemical composition of the particle and the relative 


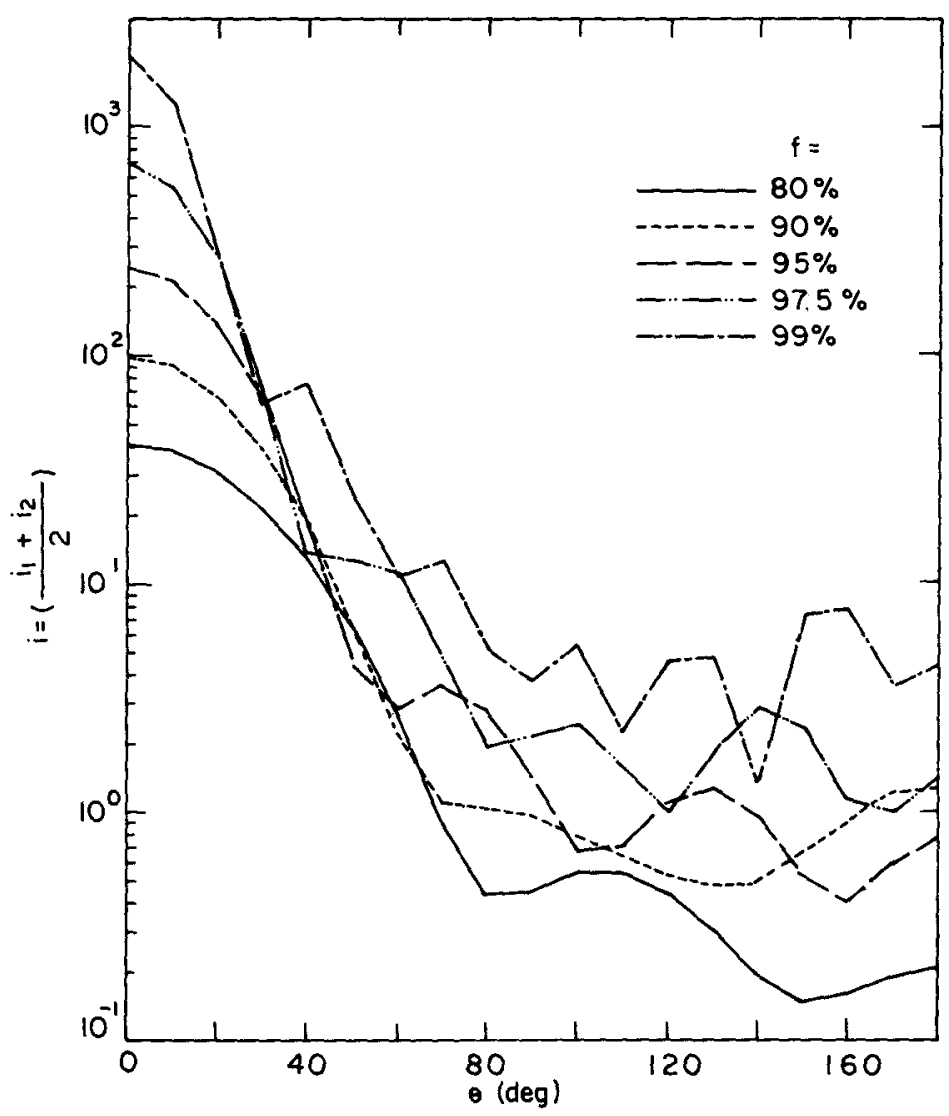

Figure 2(b). Angular variation of intensity distribution function at higher values of relative humidity $(70 \%<f \leqslant 99 \%)$ for the particle of dry radius $r(0)=0-1706 \mu \mathrm{m}$.

humidity. For the wavelength of light $\lambda=0.55 \mu \mathrm{m}$, for which the functions $i_{1}, i_{2}$ have been computed, the real as well as imaginary parts of the complex refractive index decrease and approach to those for water. McCartney (1976) has compiled the effect of each of the three parameters individually on the functions. It has been observed that for very small values of $x(<0 \cdot 2)$, the functions are symmetrical in forward and backward lobes. The angular pattern changes as $x$ increases. At higher values, the scattering is pronounced in forward lobe, especially in a small solid angle $\left(\theta<10^{\circ}\right)$ along the direction of incident beam, and fluctuates abruptly in other directions.

The real part of the refractive index (in the range of values applicable to aerosols) has modest effect on the scattering functions. It is based on the observations of McCartney (1976), who has plotted angular scattering cross-section versus the observation angle for three values of refractive index. From definition angular scattering cross-section is directly proportional to scattered intensity function and it is observed that a decrease in refractive index causes increase in the function, in forward lobe and decrease nearly everywhere else, although in a rather erratic manner. The effect of the third parameter, 


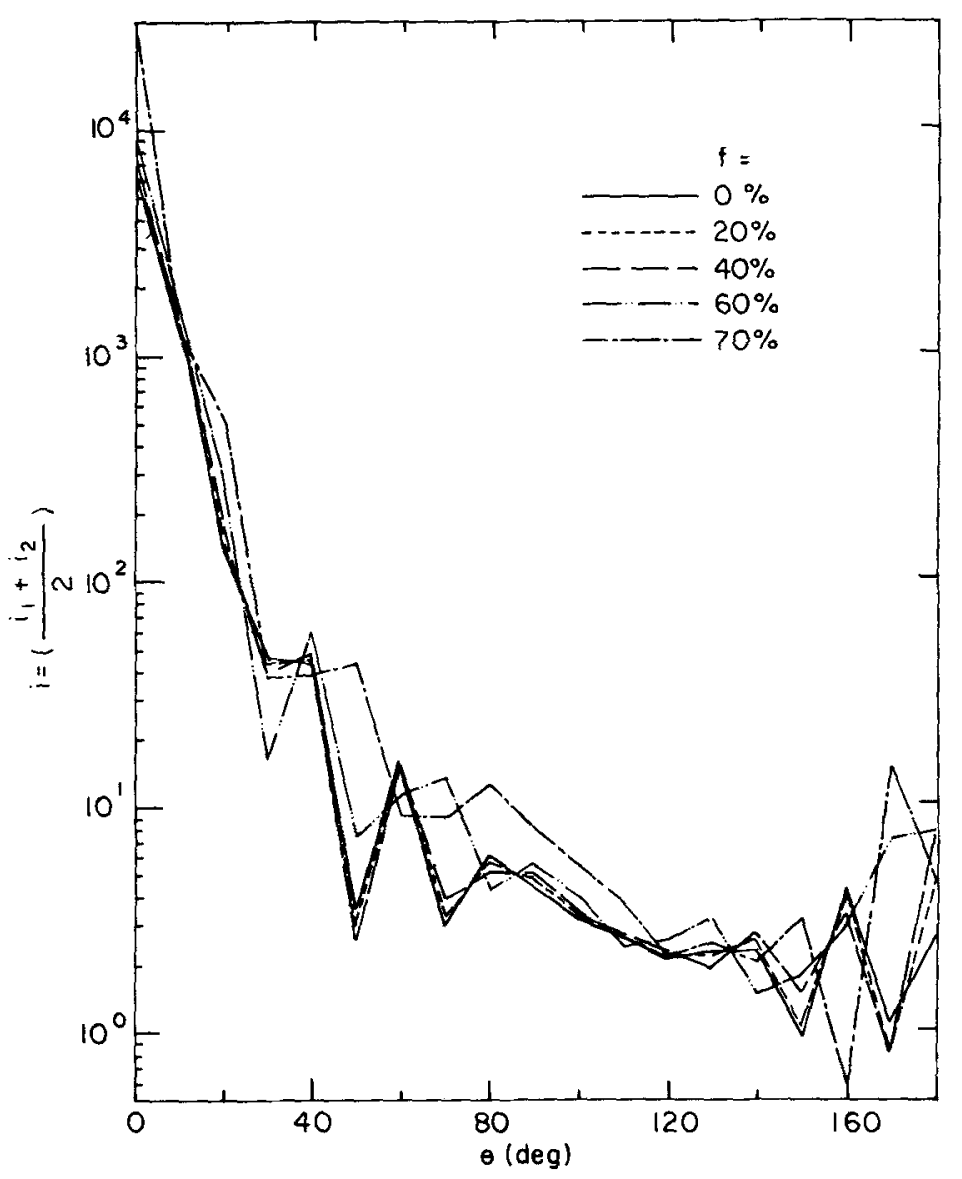

Figure 3(a). Angular variation of intensity distribution function at lower values of relative humidity $(0 \% \leqslant f \leqslant 70 \%)$ for the particle of dry radius, $r(0)=1.017 \mu \mathrm{m}$.

the imaginary part of the refractive index, on angular scattering functions has been studied by Plass (1966) and it has been observed that the ratio of back scattering $\left(180^{\circ}\right)$ to forward scattering $\left(0^{\circ}\right)$ decreases as imaginary part $(k)$ increases from zero. The ratio reaches a minimum at $10^{-2}<k<10^{-1}$ and then increases for larger values of $k$.

The angular behaviour of scattered light that we have computed here is naturally the result of all the three parameters and hence it is difficult to identify the contribution of each separately. However, from the observations and above discussion one would conclude that the size parameter dominates the effect of refractive index.

The variation in asymmetry factor for all the three particles is shown in figure 4 . The factor strongly depends on the momentum of the scattered light transmitted in the direction of incident beam and thus depends on the angular distribution of scattered light. For the particle $(r(0)=0.04472 \mu \mathrm{m})$ it is very less at lower values of relative humidity and increases sharply at higher values $(\geqslant 95 \%)$. This is in agreement with the 


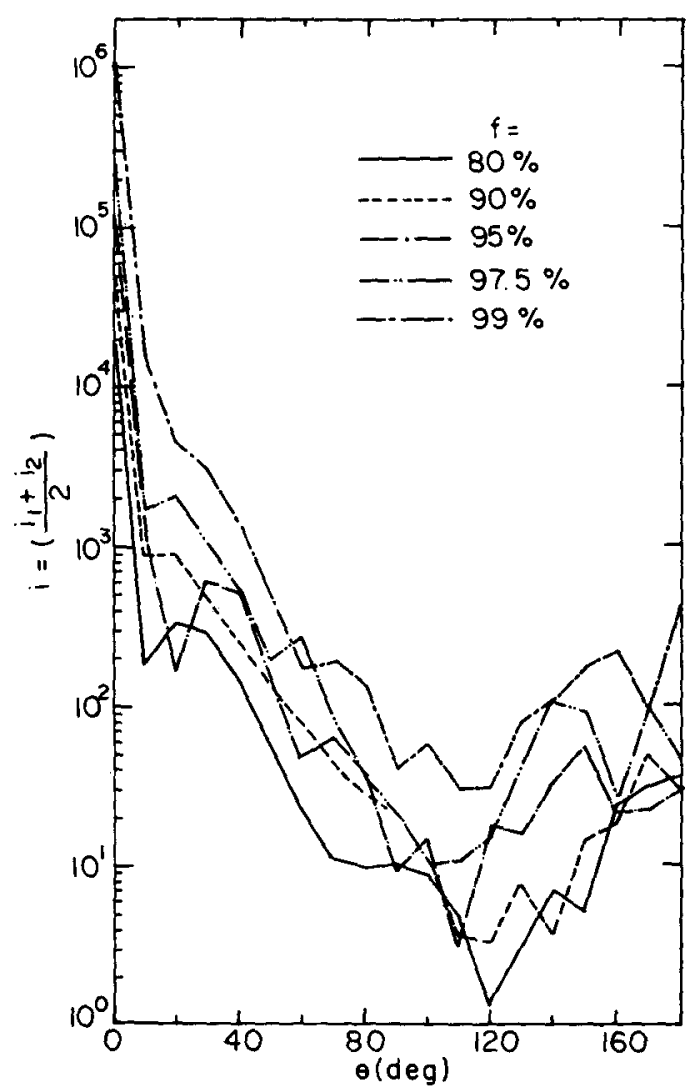

Figure 3(b). Angular variation of intensity distribution function at higher values of relative humidity $(70 \%<f \leqslant 99 \%)$ for the particle of dry radius, $r(0)=1.017 \mu \mathrm{m}$.

scattering patterns for this particle. At lower values of relative humidity, scattering is moreover uniform in forward and backward lobes and momentum in the forward direction is almost zero. At higher values, the pattern changes and scattering increases in forward direction and this causes an increase in the asymmetry factor. For the particles comparable, particularly larger to the wavelength of incident radiation, the angular pattern of the scattering function is complicated in dry conditions itself (relative humidity being zero). The increase in relative humidity complicates the scattering function and results in a rather uncertain behaviour of the asymmetry factor.

\section{Conclusion}

For three representative cases of urban aerosols, the effect of increase in relative humidity on the angular variation of scattered light intensity distribution function, $i$, has been studied theoretically. The increase in relative humidity changes the function $i$, 


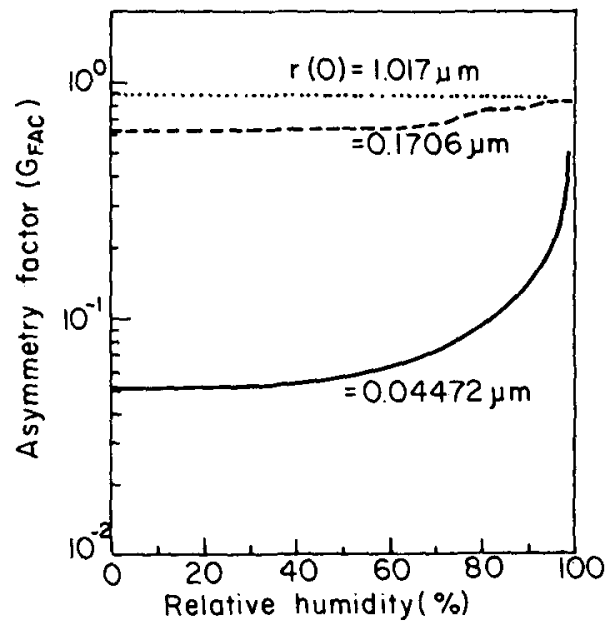

Figure 4. Variation in asymmetry factor, with relative humidity for the three particles.

through its influencing the three physical characteristics of the scatterer, namely, the size, the real part of the refractive index and the imaginary part of the refractive index. It has been found that the effect is mainly due to change in the size. For bigger particles the changes are erratic in all directions, excepting in a small solid angle about the direction of incident light. The asymmetry factor strongly increases with the relative humidity for smaller particle while for bigger particles it is more or less unaffected.

\section{Acknowledgement}

One of the authors (RKG) is thankful to University Grants Commission for financial assistance.

\section{References}

Deirmendjian D 1969 Electromagnetic scattering on spherical polydispersions (New York: American Elsevier) Denman H H 1966 Angular scattering functions for spheres (Detroit: Wayne State University Press)

Hänel G 1976 Advances in geophysics 19 (New York: Academic Press)

Kerker M 1969 The scattering of light and other electromagnetic radiation (New York: Academic Press)

Khare V 1976 Short wavelength scattering of electromagnetic waves by a homogeneous dielectric sphere, Ph.D. thesis, University of Rochester NY

McCartney E J 1976 Optics of the atmosphere (New York: John Wiley)

Plass G N 1966 Appl. Opt. 5279

Shifrin K S and Zelmanovich I L 1966 Tables of light scattering, Part I, (Lenningrad: Hydrometeorological Publishing House)

Van De Hulst H G 1957 Light scattering by small particles (New York: John Wiley)

Wiscombe W J 1979 Mie scattering calculations: Advances in technique and fast vector speed computer codes, NCAR Technical Note, NCAR/TN-140 + STR, Boulder CO. 